\title{
Long-term Effects of a Mindfulness-Based Childbirth and Parenting Program - a Randomized Controlled Trial
}

\author{
Gunilla Lönnberg $^{1}$ (1) $\cdot$ Wibke Jonas $^{1} \cdot$ Richard Bränström ${ }^{2} \cdot$ Eva Nissen $^{1} \cdot$ Maria Niemi $^{3}$
}

Published online: 13 May 2020

(C) The Author(s) 2020

\begin{abstract}
Objectives The aim of the present study was to investigate long-term effects of Mindfulness-Based Childbirth and Parenting (MBCP) during pregnancy on women's perceived stress and depressive symptoms during the first year postpartum.

Methods Women $(n=193)$ who were pregnant with their first child and at risk for perinatal depression were randomized to MBCP or an active control condition, which consisted of a Lamaze childbirth class. The women provided self-reported data on perceived stress, depressive symptoms, positive states of mind, and the Five Facets of Mindfulness Questionnaire at baseline, postintervention, and at 3,9, and 12 months postpartum.

Results Linear mixed model analysis showed that the intervention group had a larger decrease in stress $(p=0.04)$ and depression scores $(p=0.004)$ and larger increase in positive states of mind $(p<0.001)$ and mindfulness scores $(p<0.001)$ from baseline to postintervention (10-12 weeks later), compared with the active control group. These initial effects were not sustained during the follow-up period. However, analyses restricted to mothers in the MBCP condition showed that those who reported continued mindfulness practice during the follow-up period $(n=50)$ had a greater initial effect of the intervention and sustained the effects to a larger degree, compared with mothers who did not continue practicing mindfulness $(n=21)$.

Conclusions This study gives partial support for providing MBCP for pregnant women. Although the initial beneficial effects from MBCP were not sustained during the postpartum period, the findings warrant further investigations since the improvements take place in a time that is crucial for the mother-infant dyad.

Trial Registration ClinicalTrials.gov ID: NCT02441595.
\end{abstract}

Keywords Maternal $\cdot$ Pregnant $\cdot$ Mindfulness $\cdot$ Stress $\cdot$ Depression

The promotion of mental health and well-being has become a health priority within the global development agenda. By 2020 , depression is predicted to be the second leading cause of the global disease burden, and it is twice as common among

Electronic supplementary material The online version of this article (https://doi.org/10.1007/s12671-020-01403-9) contains supplementary material, which is available to authorized users.

Gunilla Lönnberg

gunilla.lonnberg@ki.se

1 Department of Women's and Children's Health, Karolinska Institutet, Solna, Sweden

2 Department of Clinical Neuroscience, Karolinska Institutet, Solna, Sweden

3 Department of Global Public Health and Center for Social Sustainability, Department of Neurobiology, Care Sciences and Society, Karolinska Institutet Sweden, Solna, Sweden women as men (WHO 2019a). Among women, pregnancy has been suggested to be a particularly vulnerable phase in life in regard to risk of mental health problems. Worldwide, the rate of women who experience a mental disorder (primarily depression) while pregnant is about $10 \%$, the corresponding number for women who have just given birth is $13 \%$ (WHO 2019b).

A growing body of evidence casts light on the intergenerational transmission of mental ill-health; perinatal stress and depression are associated with far-reaching negative psychological and developmental outcomes among children, which can last into late adolescence (Stein et al. 2014). This transmission occurs in utero as well as during the first years in life. It is thus well motivated to search for strategies that reduce maternal stress and prevent perinatal depression, not only to relieve the mother's suffering but also in order to minimize the risk of negative health effect transmission to the next generation. 
Mindfulness-based interventions (MBIs) tailored to pregnant women or expectant couples constitute a novel field of research. These programs are based on mindfulness-based stress reduction (MBSR) or mindfulness-based cognitive therapy (MBCT), with varying degrees of tailoring to fit the needs of pregnant women or expectant couples. Most programs encompass around 8 weekly sessions, and the participants are asked to practice mindfulness at home in between sessions with access to audio files with guided practices.

Mindfulness-Based Childbirth and Parenting (MBCP) is one of these MBIs, and a pilot study with 28 self-selected women found that MBCP reduced pregnancy anxiety and stress from baseline to postintervention (Duncan and Bardacke 2010). The results from pre- to postintervention measures of psychological outcomes were similar in a recent randomized control trial, in which pregnant women participating in MBCP significantly reduced perceived stress and depressive symptoms compared with an active control group (Lonnberg et al. 2019). In addition, a brief version of MBCP with 4 sessions (instead of the original 9) has proven to be feasible and acceptable, and the results also point to improvements in selfreport measures of stress and depression (Warriner et al. 2018).

Support for feasibility and indications of improvements in psychological distress was also found in a pilot study of the antenatal MBI called the MindBabyBody program (Woolhouse et al. 2014). Furthermore, Townshend et al. (2018) found reduced perinatal depression, stress, and anxiety from an 8-week mindful parenting program for pregnant women at risk of psychological distress. The latter study also indicates that antenatal MBIs promote skills development in self-kindness, observing, and acting with awareness and that these changes are associated with a reduction in perinatal depression.

In a pilot study evaluating a program called Mindful Motherhood, participants were selected based on having previously sought some form of treatment for mood disorders (Vieten and Astin 2008). Significant decreases in state anxiety and negative affect were documented, and $7-10 \%$ of these improvements were retained at a 3-month follow-up. Clinically reliable decreases in stress symptoms have also been demonstrated among pregnant women with a history of anxiety or depression (Dunn et al. 2012). In addition, Dimidjian et al. (2016) have tested MBCT for the prevention of depression relapse/recurrence among pregnant women (calling the intervention MBCT-PD) and provide evidence that MBCT-PD can prevent depression relapse among pregnant women through a follow-up period of 6 months postpartum. Similarly, lowered levels of depressive symptoms were sustained 6 months postintervention in a study by Miklowitz et al. (2015), which investigated the effects of MBCT for perinatal women with recurrent major depressive disorder or bipolar spectrum disorder.

Mindfulness-based cognitive therapy (MBCT) has also been adapted for pregnant women with general anxiety disorder in an intervention called CALM Pregnancy (Goodman et al. 2014). Goodman et al. found good feasibility and acceptability of the intervention, as well as statistically and clinically significant improvements in anxiety, depression, self-compassion, and mindfulness. In this pilot study, improvements were maintained or even further improved at the follow-up at 3 months postpartum (Luberto et al. 2018). Another pilot randomized controlled trial (RCT) testing an MBI for pregnant women with high levels of perceived stress and pregnancy anxiety also demonstrates larger decreases in anxiety from pre- to postintervention, but no sustained effects at a 6-week postintervention follow-up (Guardino et al. 2014).

Qualitative evaluations illustrate that antenatal MBIs can give participants a sense of empowerment and community (Fisher et al. 2012) and that they can be experienced as developing inner resources, self-knowledge, and self-compassion, which are found helpful during childbirth and early parenting (Lonnberg et al. 2018). Such evaluations have also elucidated that participants value the peer support established during the intervention (Dunn et al. 2012) and that antenatal mindfulness can be perceived as promoting resilience and contribute to wellbeing (Meyer et al. 2017). Furthermore, five systematic reviews of the effects of antenatal MBIs conclude that there are limited positive findings and call thus for more adequately powered, longitudinal RCTs in order to establish sufficient evidence for effectiveness (Badker and Misri 2017; Dhillon et al. 2017; Hall et al. 2016; Matvienko-Sikar et al. 2016; Shi and MacBeth 2017).

Since participants in antenatal MBIs go through childbirth and become parents after the intervention, it is of interest to evaluate if they benefit from the intervention through this transition. Sustaining self-care routines may be more challenging when also caring for an infant. Yet the benefits that may accompany continued practice of mindfulness could possibly contribute not only to less parental stress and improved mental well-being but also to the parent being more emotionally available for the infant and to positive parent-child interaction.

This longitudinal randomized controlled study, which targets first-time mothers at risk of perinatal depression, furthers the field of antenatal MBIs. We hypothesized that there may be a lasting treatment effect from MBCP on levels of stress, depressive symptoms, positive emotions, and mindfulness during the first year of motherhood. We also studied what role continued mindfulness practice after completion of the MBI program could have on long-term treatment effects.

\section{Method}

\section{Participants}

Statistical power was calculated based on perceived stress at postintervention and data from a pilot study by Duncan and 
Bardacke (2010). Between 2014 and 2016, women who expected their first child and received maternal health care checkups at one of eight different maternal health clinics in Stockholm County were invited to participate in the study. Around gestational week 15-22 of pregnancy, the women received a letter of invitation to the study, with information regarding the study and a short description of MBCP and Lamaze. In order to keep participants blinded to our hypotheses, the aim in the letter was expressed as "to study parental support, stress and quality of life, by comparing two different courses." Partners were encouraged to participate in the antenatal classes as well. Single women and women with a partner who could not participate were welcome to attend by themselves or in the company of a support person.

Women who were interested in participating were asked to fill out a brief online questionnaire to assess eligibility. To be eligible, the pregnant woman should be at risk for perinatal depression. Therefore, we selected women with a history of depression or anxiety, early life adversity, and/or current high levels of perceived stress. The inclusion criteria included at least one of the following criteria (a-d): (a) scoring six points or higher on the 4-item Perceived Stress Scale (Cohen et al. 1983), (b) having previously sought health care for mental health problems (yes/no and if yes, they were asked to describe what kind and when), (c) previous experience of depression or anxiety (yes/no and if yes, they were asked to describe what kind and when), or (d) scoring six points or higher on three selected items from the Childhood Trauma Questionnaire (Bernstein et al. 2003) using a 5-point scale from 0 to 4 with a high score indicating early life adversity. In addition, the participant should be (e) fluent in Swedish, (f) have had no previous experience of mindfulness training or meditation, (g) no current psychotic symptoms or major depressive episode (given the focus on prevention, not on acute intervention), and (h) be at least 18 years old.

Figure 1 describes the sample size throughout all study phases, including exclusion, ineligibility, and dropouts. In all, 1647 letters were sent to invite women for screening. A total of 347 women were assessed for eligibility, and 193 met the inclusion criteria and agreed to participate; 96 were randomized to the MBCP group and 97 to the Lamaze group (Fig. 1). Eighty-six percent completed the postintervention assessment; $79.27 \%$ completed the 3-month assessments; $73.36 \%$ completed the 9 -month assessment; and $68.39 \%$ completed the 12-month assessment.

Table 1 presents the socio-demographic background and inclusion criteria of the participating women. Several participants met multiple inclusion criteria; when combining the criteria regarding perceived stress, early life adversity, and previously having sought healthcare for mental health problems, about half of the participants in both study arms met two of these criteria and about a quarter in each arm met all three of these criteria. The majority of women was highly educated and lived with a partner. There were no significant differences in socio-demographic background, inclusion criteria, or scores on the baseline questionnaires between those allocated to the MBCP group and the Lamaze group. Thus, these data were not included in our further analyses. There were no significant differences between completers and dropouts on sociodemographic background variables or scores on the baseline questionnaires (data not shown).

Four women in the MBCP arm and three women in the Lamaze arm gave birth prematurely, between gestational weeks 32 and 36. In the MBCP arm, $62 \%$ of the infants born were boys, $38 \%$ were girls, and one mother gave birth to twins. In the Lamaze arm, $39 \%$ of the infants were boys and $61 \%$ were girls and three mothers gave birth to twins.

One adverse event was reported; a woman in the MBCP arm experienced increased anxiety during the group meetings and dropped out.

\section{Procedure}

Eligible women were scheduled for an appointment where baseline assessments were completed and during which they signed informed consent to participate. After completion of the baseline questionnaires, an administrator who was not part of the research team randomized the participants to either intervention or active control, by using a randomization sequence generated in SPSS in blocks of ten. Postintervention assessment was carried out 10 to 12 weeks after the baseline assessment, and subsequent follow-up assessments were carried out at 3, 9, and 12 months postpartum.

Intervention Condition-MBCP The MBCP program was an adapted and culturally adjusted version of a program developed by Bardacke (2012). The adaptations and details of the program content are described in greater detail in our previous publication (Lonnberg et al. 2019) and are also provided as supplementary materials. The program consisted of eight weekly group-based sessions, each $2 \mathrm{~h}$ and $15 \mathrm{~min}$ long, in total 18 contact hours, during which antenatal education was interwoven with mindfulness practices such as body scan, sitting and walking meditation, mindful movement, loving kindness meditation, and informal meditation in daily life. Moreover, the program included mindfulness practices specific for MBCP such as mindful speaking and listening inquiry between the couples, methods to increase awareness of the baby, and how to cope with pain during labor. A 15-min snack break during each session served as an opportunity for interaction between group participants.

An average of 8-9 women were included in each group, and most women $(89 \%)$ were accompanied by their partner during the sessions. At the start of the intervention, the gestational ages of the pregnant women were between 19 and 
Fig. 1 Flowchart of participants (CONSORT figure)

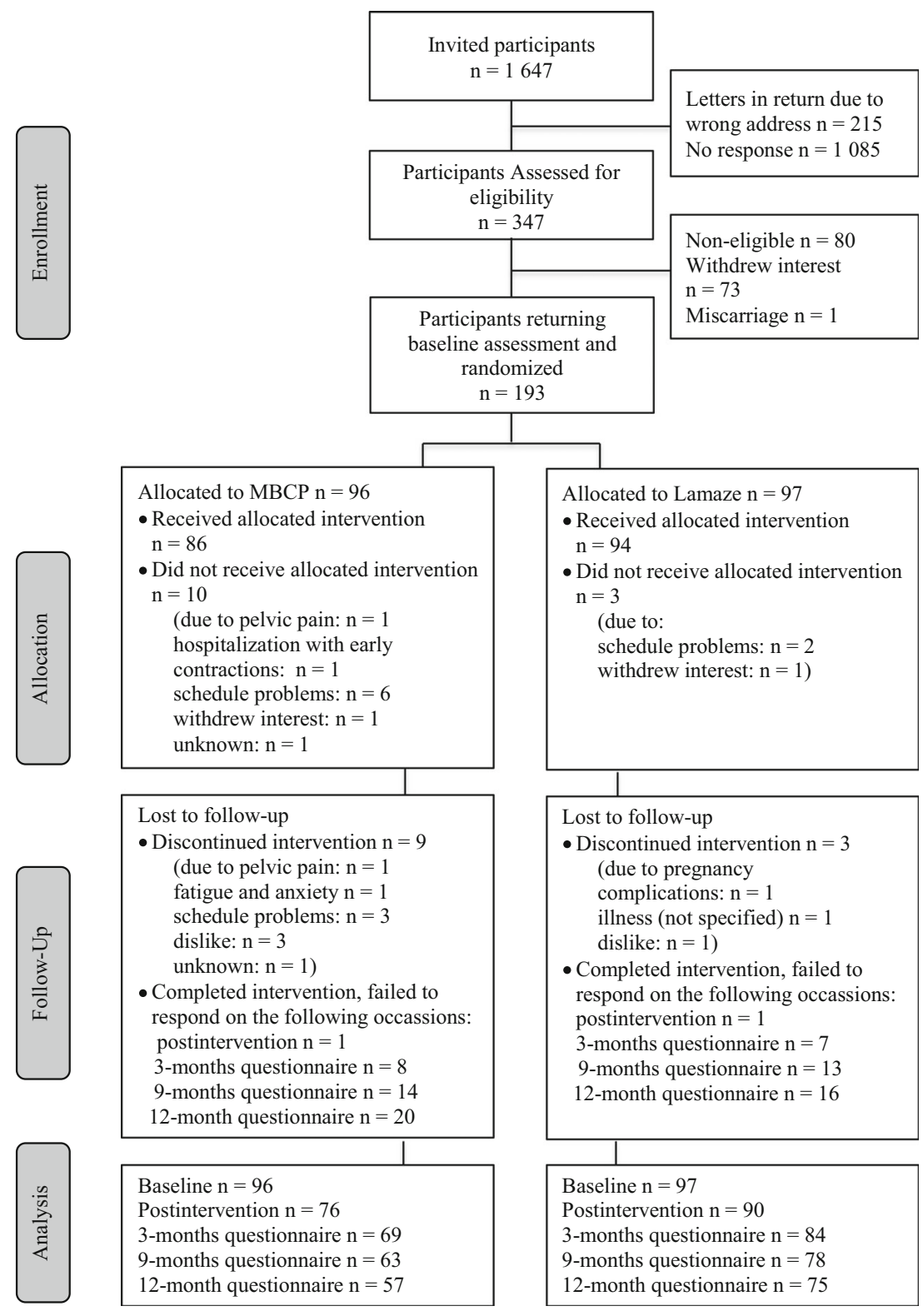

26 weeks and at the end between 27 and 34 weeks. Between sessions, home assignments were encouraged. Homeassignments consisted of formal mindfulness practice, $30 \mathrm{~min}$ per day throughout the program, as well as informal practice. Participants were encouraged to perform informal mindfulness practice whenever they sensed fetal movements and during other daily activities (e.g., while taking a shower, brushing teeth, or preparing food). The participants had access to audio files with guided formal mindfulness practices throughout the program. Participants' experiences of practicing mindfulness were discussed within the group during each session. All groups had a scheduled reunion within 24 months after the birth of their babies.

Three teachers, all graduates from a teacher training in MBCP (Bardacke 2019) and with at least 10 years of meditation experience, delivered the intervention. Frequent meetings were held between the teachers to discuss their teaching and ensure fidelity to the intervention. The teachers were not blinded to the experimental hypothesis. In the case of adverse events, the teachers were instructed to report them to the research team.

Active Control Condition-Lamaze Childbirth Program To control for possible effects of social support and psychoeducation, the Lamaze program (Frisk 2018) was chosen as the active control. This choice also facilitated feasibility of the study since this program is widely available in Stockholm and its childbirth preparation component is appreciated by the majority of participating women and men (Bergström et al. 2011). 
Table 1 Socio-economic background characteristics and inclusion criteria of all participants $(n=193)$. Presented with means and standard deviations (SD). $t$ tests, Chi-square test, and Fisher's exact test were performed

\begin{tabular}{|c|c|c|c|c|c|}
\hline Variable & $\begin{array}{l}\text { MBCP }(n=96) \\
\text { Mean }(\mathrm{SD})\end{array}$ & $\begin{array}{l}\text { Lamaze }(n=97) \\
\text { Mean }(\mathrm{SD})\end{array}$ & Condition comparisons & $d f$ & $p$ \\
\hline Age (years) & $32(3.86)$ & $32(4.14)$ & $t=-0.52$ & 191 & 0.602 \\
\hline Civil status & $n(\%)$ & $n(\%)$ & $F E T=2.102$ & 3 & 0.607 \\
\hline Single & $3(3.1 \%)$ & $2(2.1 \%)$ & & & \\
\hline Co-living & $57(59.4 \%)$ & $60(61.7 \%)$ & & & \\
\hline Married & $36(37.5 \%)$ & $33(34.0 \%)$ & & & \\
\hline Living apart & $0(0 \%)$ & $2(2.1 \%)$ & & & \\
\hline Nationality & & & $F E T=1.267$ & 3 & 0.776 \\
\hline Swedish & $86(89.6 \%)$ & $83(85.6 \%)$ & & & \\
\hline Swedish \& other & $3(3.1 \%)$ & $6(6.2 \%)$ & & & \\
\hline European & $5(5.2 \%)$ & $6(6.2 \%)$ & & & \\
\hline Non-European & $2(2.1 \%)$ & $2(2.1 \%)$ & & & \\
\hline Education $^{\mathrm{a}}$ & & & $F E T=2.579$ & 3 & 0.636 \\
\hline Elementary & $1(1.0 \%)$ & $0(0 \%)$ & & & \\
\hline Secondary & $12(12.5 \%)$ & $12(12.6 \%)$ & & & \\
\hline College & $83(86.5 \%)$ & $83(86.6 \%)$ & & & \\
\hline Work hours ${ }^{\mathrm{b}}$ & & & $X^{2}=5.199$ & 3 & 0.158 \\
\hline Up to $40 \mathrm{~h} /$ week & $69(72.6 \%)$ & $70(72.2 \%)$ & & & \\
\hline More than $40 \mathrm{~h} /$ week & $26(27.4 \%)$ & $27(27.8 \%)$ & & & \\
\hline Household income/month ${ }^{\mathrm{a}}$ & & & $F E T=4.247$ & 4 & 0.345 \\
\hline$<25,000 \mathrm{SEK}$ & $2(2.2 \%)$ & $0(0 \%)$ & & & \\
\hline 25-40,000 SEK & $15(16.0 \%)$ & $14(14.4 \%)$ & & & \\
\hline 40-60,000 SEK & $30(31.9 \%)$ & $27(27.8 \%)$ & & & \\
\hline$>60,000 \mathrm{SEK}$ & $47(50.0 \%)$ & $56(57.7 \%)$ & & & \\
\hline Prescribed drug use & & & $F E T=2.71$ & 4 & 0.589 \\
\hline None & $66(68.8 \%)$ & $72(74.2 \%)$ & & & \\
\hline ADHD medication & $0(0 \%)$ & $1(1.0 \%)$ & & & \\
\hline SSRI medication & $8(8.3 \%)$ & $5(5.2 \%)$ & & & \\
\hline Sedatives & $1(1.0 \%)$ & $2(2.1 \%)$ & & & \\
\hline Non-psychotropic & $21(21.9 \%)$ & $17(17.5 \%)$ & & & \\
\hline \multicolumn{6}{|l|}{ Inclusion criteria $^{a}$} \\
\hline$>6$ on perceived stress & $69(72.6 \%)$ & $75(78.1 \%)$ & $X^{2}=0.777$ & 1 & 0.378 \\
\hline$>6$ on childhood trauma & $42(44.2 \%)$ & $33(34.4 \%)$ & $X^{2}=1.937$ & 1 & 0.164 \\
\hline Previous mental healthcare & $65(68.4 \%)$ & $61(63.5 \%)$ & $X^{2}=0.506$ & 1 & 0.477 \\
\hline Previous depression/anxiety & $80(85.1 \%)$ & $73(76.0 \%)$ & $X^{2}=2.489$ & 1 & 0.115 \\
\hline
\end{tabular}

FET Fisher's exact test

${ }^{\text {a }}$ Data is missing for two participants

${ }^{\mathrm{b}}$ Data is missing for one participant

During three weekly sessions, each 3 hours long, in total 9 contact hours, the participants received instructions and training in breathing and relaxation techniques and mental training exercises, and partners/support persons were given instructions on how to support the pregnant woman during labor. Participants also learned about, e.g., breastfeeding and life with a newborn. At the start of the intervention, the gestational ages of the pregnant women were between 24 and 31 weeks and at the end between 27 and 34 weeks.

Three different teachers taught the program, all of whom were trained and experienced in teaching the Lamaze childbirth program. The teachers were not blinded to the experimental hypothesis. The Lamaze sessions started approximately 5 weeks after the start of the MBCP sessions so that both 
courses finished about the same time before the postintervention assessment.

\section{Measures}

PSS The Perceived Stress Scale (PSS) is used to assess the frequency of stressful experiences during the past month and consists of fourteen items (Cohen et al. 1983). Responses are indicated on a 5-point scale, ranging from 0 ("never") to 4 ("very often") and scores range from 0 to 56 , with higher scores indicating greater perceived stress. In this study, a validated Swedish translation was used (Eklund et al. 2014).

EPDS The Edinburgh Postnatal Depression Scale (EPDS) is used to assess the severity of depressive symptoms during the past week with ten items (Cox et al. 1987). In Sweden, the EPDS is widely used as a screening tool to identify mothers at risk for postpartum depression. Items are scored on a fourpoint scale ranging from 0 to 3 , and total scores range from 0 to 30 , with higher scores indicating more severe depressive symptoms. The EPDS is frequently used and a translation to Swedish has been validated (Rubertsson et al. 2011). In the main analysis, EPDS was used as a continuous measure. However, in addition to the main analyses, a cut-off set at 11/12 for detection of depressive symptoms was used to compare number of women with elevated scores in the two groups. This cut-off has been demonstrated to have a sensitivity of 96\% and a specificity of $49 \%$ (Wickberg and Hwang 1996).

In order to complement our main outcomes-perceived stress and depressive symptoms - and also to gain an understanding of health promoting aspects that may build inner resources (Fredrickson 2001), we added an outcome related to positive affect, as well as an outcome attempting to measure mindfulness, described below:

PSOM The Positive States of Mind (PSOM) has six items and is used to measure positive experiences regarding focused attention, productivity, responsible caretaking, restful repose, sharing, and sensuous nonsexual pleasure (Adler et al. 1998; Horowitz et al. 1988). Scores range from 5 to 30, where high scores indicate a high capacity to experience positive states of mind.

FFMQ We used the Swedish Version of the Five-Facet Mindfulness Questionnaire (FFMQ), which has 29 items that measure five factors representing elements of mindfulness (Lilja et al. 2011). Items are rated on a 5-point Likert scale ranging from 1 ("never/almost never") to 5 ("always"). Scores range from 29 to 145 and can be divided into sub-scores for the following facets; nonreactivity to inner experience, observing, acting with awareness, describing and non-judging of inner experience (Baer et al. 2006). High scores indicate a higher capacity to be mindful.
Internal consistency was high for all four measures in this study (Cronbach alpha for PSS $=.82$, for $\mathrm{EPDS}=.85$, for $\mathrm{PSOM}=.83$, for $\mathrm{FFMQ}=.85$ ), and all four measures were used as continuous variables in the analyses.

Continued Practice during the Follow-Up Period At the follow-up assessments at 3 months, 6 months, and 12 months postpartum, the participants in the MBCP condition filled out a form regarding how often and for how long they had been practicing formal and informal mindfulness meditation on average during the last month. In the analysis, continued practice was used both as a continuous variable and as a dichotomous variable, either none or some continued practice.

\section{Data Analyses}

Analyses were conducted with an intention-to-treat approach, and we used Linear Mixed Model (LMM) analyses in SPSS (Version 25) to assess differences between the intervention and control groups at postintervention and at 3-, 6-, and 12month follow-up assessments. The LMM uses all available data points and thus minimizes information loss due to missing data (Hesser 2015). Maximum likelihood was used as the method of estimation. The fixed effect interaction terms between group and time were the parameters of main interest, in order to describe whether women in the two groups showed differences in change in stress levels and depressive symptoms over time. Random intercepts and slopes were added in the models one at a time and turned out to improve model fit. The covariance structure used was "Variance Components," and this was chosen by fitting models with competing covariance structures and choosing the best fitting model. First, a model was run with the dependent variables PSS and EPDS, one at a time, with time, group, and group $\times$ time interaction. This was repeated with the secondary outcome variables PSOM and FFMQ.

To accommodate for nonlinear change over time, we also studied the difference in change over time between the two groups using two distinct time periods, i.e., change between baseline and postintervention and change from postintervention and across the three follow-up assessments. Thus, we constructed a piecewise growth model with two time-pieces: The first time period represents the changes from baseline to postintervention (time-piece 1), and the second time period represents the changes during the follow-up period from postintervention to 12 months postpartum (time-piece 2). To examine the difference in change over time between the groups, we included the interaction terms for both time-pieces (i.e., group $\times$ time-piece 1 and group $\times$ time-piece 2 ).

To explore the effect of continued practice on the effect of the MBCP, additional subgroup analyses were performed. In this subgroup analysis among the mothers allocated to MBCP, the same piecewise growth model was run to investigate if 


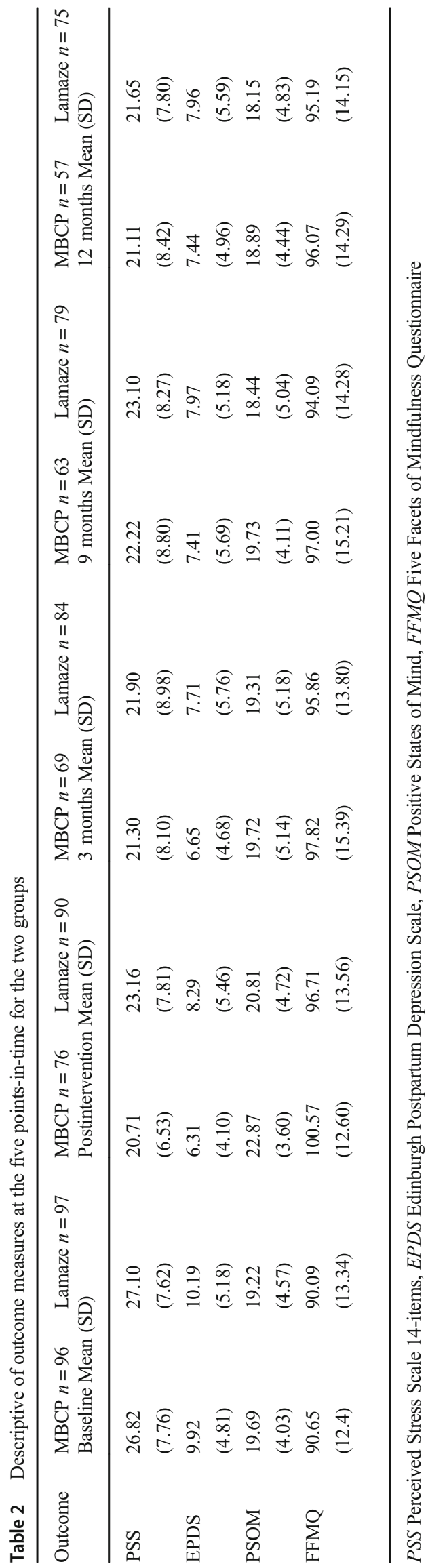

there were differences in change over time as a function of continued mindfulness practice during the follow-up period. Continued practice was tested both as a continuous variable and as a dichotomous variable.

\section{Results}

\section{Treatment Effects on Stress and Depressive Symptoms}

The means and standard deviations (SD) of scores on PSS and EPDS at baseline, postintervention, and at 3, 9, and 12 months postpartum are presented in Table 2. Correlations between the four measures at baseline are shown in the supplementary material.

Mean scores across all measurement points are illustrated separately for intervention and control group in Fig. 2.

The first model including all time-points showed a significant change over time (PSS F $=11.90, p<0.001$, EPDS F $=$ $5.92, p<0.001)$ indicating that both groups improved from baseline to the 1-year follow-up. There were no statistically significant interaction effects between group and time (PSS $\mathrm{F}=1.01, p=0.40 ; \mathrm{EPDS} \mathrm{F}=1.70, p=0.15$ ), indicating a similar development of stress and depression trajectories in both groups.

The test of group differences during two specific time periods (i.e., pre- to postintervention and postintervention across 3-, 6-, and 12-month follow-ups), conducted with the piecewise growth model, showed that both groups significantly decreased their PSS scores from baseline to postintervention $(\mathrm{F}=23.80 p<0.001)$, and there was a group $\times$ time interaction in PSS scores $(\mathrm{F}=4.22 p=0.04)$ showing that the decrease in PSS scores was larger in the intervention group than in the control group. However, from postintervention through 12month follow-up, neither group had any significant change $(\mathrm{F}=1.79, p=0.15)$, and there was no significant group $\times$ time interaction $(\mathrm{F}=1.30, p=0.27)$.

A similar pattern was found for EPDS. The piecewise growth models showed that both groups significantly decreased their EPDS scores from baseline to postintervention $(\mathrm{F}=15.12, p<0.001)$, and there was a significant group $\times$ time interaction $(\mathrm{F}=8.52, p=0.004)$, where the decrease in EPDS scores was larger in the intervention group than in the control group. From postintervention to 12 months postpartum, the groups had no significant change $(\mathrm{F}=0.57, p=0.63)$ and the group $\times$ time interaction was not significant $(\mathrm{F}=1.86$, $p=0.13$ ).

Regarding the number of women who had elevated scores on EPDS at the five points-in-time, at baseline, $39.6 \%$ of the MBCP-mothers and $33.0 \%$ of the Lamaze-mothers scored above the EPDS cut-off $(p=.341)$. The corresponding rates were at postintervention: $10.7 \%_{\mathrm{MBCP}}$ and $23.3 \%_{\text {Lamaze }}$ 


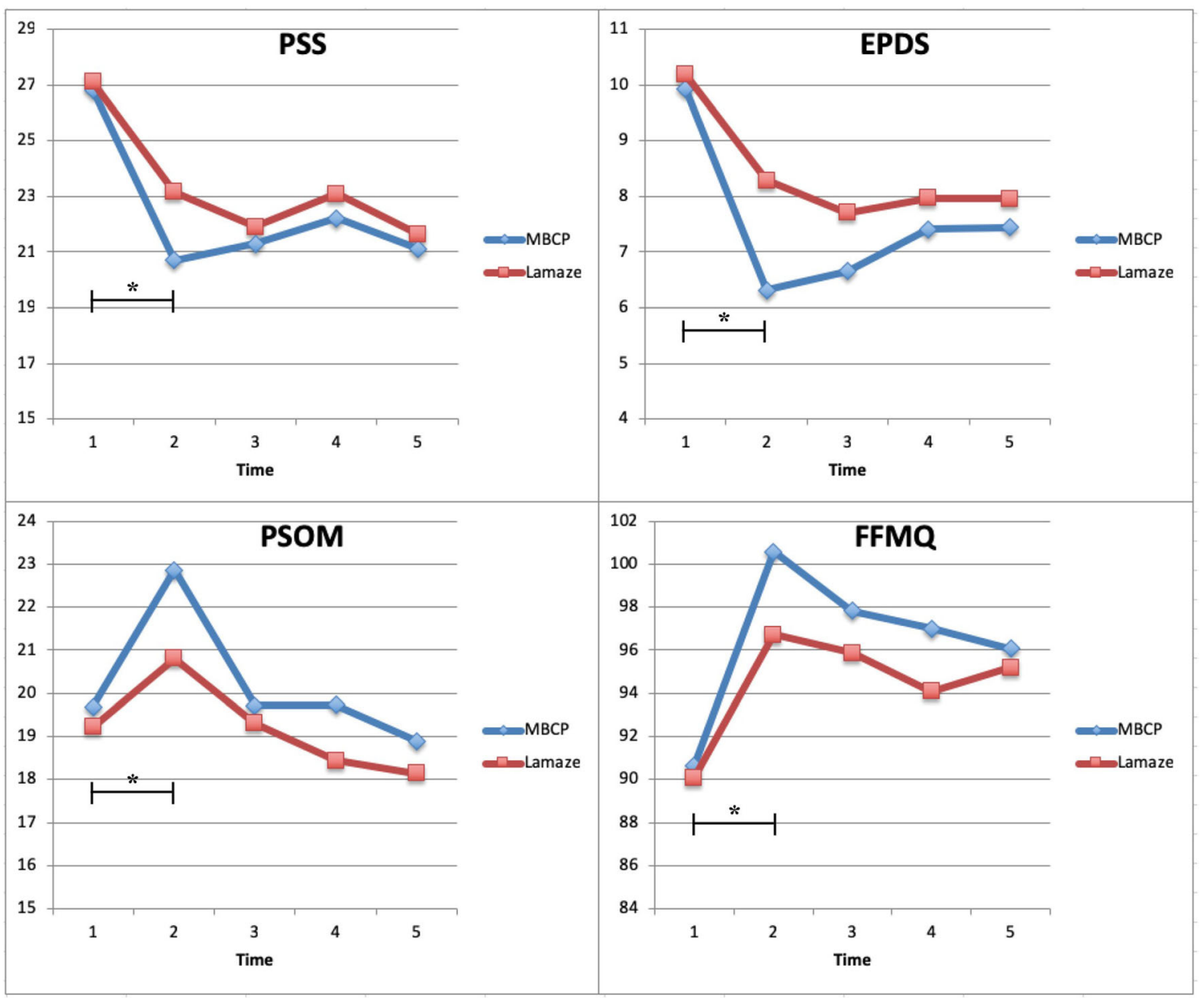

Fig. 2 Change over time for the two groups MBCP and Lamaze for the outcomes PSS (Perceived Stress Scale 14-items), EPDS (Edinburgh Postpartum Depression Scale), PSOM (Positive States of Mind), and FFMQ (Five Facets of Mindfulness Questionnaire). $X$-axis: $1=$

$(p=.033)$; at 3 months postpartum: $14.5 \%_{\mathrm{MBCP}}$ and $22.6 \%_{\text {Lamaze }}(p=.202)$; at 9 months postpartum: $20.6 \%_{\mathrm{MBCP}}$ and $25.6 \%$ Lamaze $(p=.485)$; and at 12 months postpartum: $15.8 \%_{\mathrm{MBCP}}$ and $30.7 \%_{\text {Lamaze }}(p=.048)$.

\section{Treatment Effects on Positive States of Mind and Mindfulness}

The secondary outcomes PSOM and FFMQ followed similar patterns as the main outcomes (see Table 3 for means and SD at the five time-points and Fig. 2 for mean score trajectories). The first model including all time-points showed a significant improvement in scores over time (PSOM F $=7.10, p<0.001$, FFMQ F $=8.35 p<0.001$, and there were no statistically significant interaction effects between group and time (PSOM $\mathrm{F}=1.75, p=0.14$, FFMQ F $=2.41, p=0.06$ ).

The piecewise model showed that from baseline to postintervention, there was a significant change in both groups $(\mathrm{F}=$ $11.65, p=0.001)$ and a significant group $\times$ time interaction with a larger increase in PSOM score for the intervention baseline, $2=$ postintervention (before childbirth), $3=3$ months postpartum, $4=9$ months postpartum, $5=1$ year postpartum. Asterisk $(*)=$ region where the group $\times$ time interaction was significant

group compared with the control group $(\mathrm{F}=6.97, p=0.01)$. During the follow-up period from postintervention to 12 months postpartum, both groups decreased their PSOM scores $(\mathrm{F}=9.82, p<0.001)$ and there was no significant difference between the groups $(\mathrm{F}=1.82 p=0.14)$.

Regarding the FFMQ scores, there was a significant change in both groups from pre- to postintervention $(\mathrm{F}=$ $39.22, p<0.001)$ and a significant group $\times$ time interaction ( $\mathrm{F}=6.80 p=0.01$ ) where mothers in the intervention group showed a larger increase in FFMQ score compared with the control. During the follow-up period, there was no significant change in the two groups $(\mathrm{F}=1.95, p=0.12)$ and no significant difference between the groups $(\mathrm{F}=2.30 p=0.08)$.

\section{Continued Practice}

Among the mothers who had participated in the MBCP program, there was a wide spread in the amount of mindfulness practice they had engaged in. During the 8 -week intervention, the mean amount of formal practice was $62.9(\mathrm{SD}=47.3) \mathrm{min}$ 
Table 3 Descriptive of outcome measures at the five points-in-time for the MBCP mothers who continued to practice mindfulness (Yes) compared with mothers who did not (No)

\begin{tabular}{|c|c|c|c|c|c|c|c|c|c|c|}
\hline \multirow[t]{2}{*}{ Group $m(S D)$} & \multicolumn{2}{|l|}{ Baseline } & \multicolumn{2}{|c|}{ Postintervention } & \multicolumn{2}{|l|}{3 months } & \multicolumn{2}{|l|}{9 months } & \multicolumn{2}{|l|}{12 months } \\
\hline & Yes $n=50$ & No $n=21$ & Yes $n=49$ & No $n=20$ & Yes $n=48$ & No $n=20$ & Yes $n=46$ & No $n=17$ & Yes $n=41$ & No $n=16$ \\
\hline PSS & $\begin{array}{l}27.30 \\
(8.35)\end{array}$ & $\begin{array}{l}25.86 \\
(7.26)\end{array}$ & $\begin{array}{l}19.71 \\
(6.50)\end{array}$ & $\begin{array}{l}22.50 \\
(6.24)\end{array}$ & $\begin{array}{l}19.50 \\
(7.73)\end{array}$ & $\begin{array}{l}25.55 \\
(7.72)\end{array}$ & $\begin{array}{l}20.98 \\
(9.09)\end{array}$ & $\begin{array}{l}25.59 \\
(7.14)\end{array}$ & $\begin{array}{l}20.29 \\
(8.79)\end{array}$ & $\begin{array}{l}23.19 \\
(7.41)\end{array}$ \\
\hline EPDS & $\begin{array}{l}10.26 \\
(5.09)\end{array}$ & $\begin{array}{l}8.67 \\
(4.11)\end{array}$ & $\begin{array}{l}6.04 \\
(4.12)\end{array}$ & $\begin{array}{l}6.10 \\
(3.67)\end{array}$ & $\begin{array}{l}5.23 \\
(3.58)\end{array}$ & $\begin{array}{l}9.95 \\
(5.46)\end{array}$ & $\begin{array}{l}6.83 \\
(5.52)\end{array}$ & $\begin{array}{l}9.00 \\
(6.00)\end{array}$ & $\begin{array}{l}6.54 \\
(4.40)\end{array}$ & $\begin{array}{l}9.75 \\
(5.67)\end{array}$ \\
\hline PSOM & $\begin{array}{l}19.04 \\
(4.16)\end{array}$ & $\begin{array}{l}19.95 \\
(4.09)\end{array}$ & $\begin{array}{l}23.45 \\
(3.23)\end{array}$ & $\begin{array}{l}21.75 \\
(4.22)\end{array}$ & $\begin{array}{l}21.00 \\
(4.71)\end{array}$ & $\begin{array}{l}16.75 \\
(5.13)\end{array}$ & $\begin{array}{l}20.09 \\
(3.53)\end{array}$ & $\begin{array}{l}18.76 \\
(5.40)\end{array}$ & $\begin{array}{l}19.32 \\
(4.50)\end{array}$ & $\begin{array}{l}17.81 \\
(4.23)\end{array}$ \\
\hline FFMQ & $\begin{array}{l}91.14 \\
(12.63)\end{array}$ & $\begin{array}{l}87.19 \\
(11.61)\end{array}$ & $\begin{array}{l}103.50 \\
(10.58)\end{array}$ & $\begin{array}{l}94.90 \\
(15.71)\end{array}$ & $\begin{array}{l}102.27 \\
(14.19)\end{array}$ & $\begin{array}{l}86.58 \\
(13.05)\end{array}$ & $\begin{array}{l}101.24 \\
(13.15)\end{array}$ & $\begin{array}{l}85.53 \\
(14.79)\end{array}$ & $\begin{array}{l}99.98 \\
(10.86)\end{array}$ & $\begin{array}{l}86.06 \\
(17.29)\end{array}$ \\
\hline
\end{tabular}

PSS Perceived Stress Scale 14-items, EPDS Edinburgh Postpartum Depression Scale, PSOM Positive States of Mind, FFMQ Five Facets of Mindfulness Questionnaire

per week and for informal practice the mean was $42.0(\mathrm{SD}=$ 44.1) min per week. After the course, the amount of practice decreased over time. At 3 months postpartum, the mean of formal practice was $10.5(\mathrm{SD}=28.9) \mathrm{min}$ per week and of informal practice it was $20.0(\mathrm{SD}=36.8) \mathrm{min}$ per week. The decrease in practice continued at 9- and 12-month follow-ups, with both less reported formal practice $\left(\mathrm{Mean}_{9 \text {-month }}=11.6\right.$, $\mathrm{SD}=23.7$; Mean $_{12 \text {-month }}=8.0, \mathrm{SD}=20.2$ ) and informal practice $\left(\mathrm{Mean}_{9 \text {-month }}=16.1, \mathrm{SD}=36.0 ; \mathrm{Mean}_{12-\text { month }}=10.2\right.$, $\mathrm{SD}=25.5$ ).

The piecewise growth LMM analysis with continued practice as a continuous variable showed no significant dosage effect from the reported amount of practice. However, the piecewise growth LMM analysis with continued practice as a dichotomous variable comparing "continuers" with "noncontinuers" showed that from pre- to postintervention, the continuers had a significantly larger decrease in PSS scores $(\mathrm{F}=6.39, p=0.014)$ and increase in PSOM scores $(\mathrm{F}=4.67$, $p=0.034)$ compared with the non-continuers. In the second time period, from postintervention through 12 months postpartum, there were no statistically significant differences in the measures of perceived stress and positive states of mind. There were no significant differences in the baseline measures or sociodemographic data between the "continuers" $(n=50$, $70 \%)$ and the "non-continuers" $(n=21,30 \%)$.

In the measures EPDS and FFMQ, there were no statistically significant differences between the two subgroups from pre- to postintervention. However, in the second time period, from postintervention to 12 months postpartum, there was a significantly larger increase in EPDS score $(\mathrm{F}=3.63, p=$ $0.014)$ and decrease in FFMQ score $(\mathrm{F}=3.32, p=0.021)$ among the non-continuers compared with the continuers.

The means and standard deviations of scores on PSS, EPDS, PSOM, and FFMQ at baseline, postintervention, and at 3,9 , and 12 months postpartum for these two subgroups within the MBCP-arm are presented in Table 3.

\section{Discussion}

The results in this study extend findings from our previous publication reporting significant improvements from pre- to postintervention in perceived stress, depressive symptoms, positive states of mind, and mindfulness among pregnant women participating in a MBCP program compared with those participating in an active control condition (Lonnberg et al. 2019). In the present study, we found that these effects were not sustained at longer-term follow-up assessments at 3 , 9 , and 12 months postpartum. Among the mothers in the $\mathrm{MBCP}$ arm, the majority (70\%) continued to practice mindfulness through the follow-up period. These mothers had a significantly larger treatment effect from pre- to postpartum compared with the mothers who did not continue to practice. They also retained the positive treatment effects to a significantly higher degree through the follow-up period.

In accordance with the MBCP-curriculum, our participants were encouraged to keep practicing mindfulness. They were advised that when they become parents, they may not find time for formal meditation, but plenty of time for informal meditation with the baby. In line with this advice, we observed a shift in the ratio of practices, to less formal and more informal practice at postpartum.

To our knowledge, the role of continued practice of mindfulness among postpartum mothers has only been tested previously in a study with a small sample size $(n=20)$ (Luberto et al. 2018). In contrast to our results, Luberto et al. did not find any significant differences in psychological outcomes between mothers who were and were not still practicing mindfulness, apart from a trend for less worry among the mothers who still practiced. However, this analysis was underpowered due to the small sample size.

Our findings regarding the role of continued practice indicate that the dose effect is not linear, but rather that there seems to be a threshold effect between no practice at all and a small 
amount of practice. A factor that possibly contributes to whether a participant continues to practice or not could be the extent to which they experienced the intervention as helpful. Among our MBCP participants, the women who continued to practice had significantly greater reductions in perceived stress and increases in positive states of mind at postintervention compared with the women who did not continue to practice, which supports the hypothesis. Indeed, this hypothesis is also supported by qualitative data from participants in MBCP, which reveals that one of the common hindrances to practicing mindfulness at home in between sessions is a sense of doubt that it could be helpful and that this prevents participants from engaging more fully during the course (Lonnberg et al. 2018).

Other studies have given further possible explanations that were not assessed in the present study, for differences among participants in adherence to MBI programs and continued practice during follow-up periods: For example, personality dimensions such as openness to experience and agreeableness have been shown to predict greater use of an MBI both during and after the intervention and may explain the variations in postintervention use of mindfulness practices, both directly and by fostering initial engagement in practice during the intervention (Barkan et al. 2016). There are also indications that different neurobiological profiles can predispose individuals to engage more or less with meditation techniques, which may, in turn, predict the magnitude of change effected by MBIs (Mascaro et al. 2013). Therefore, it is unclear if our results regarding "continuers" vs "non-continuers" reflect a specific effect of mindfulness practice or a more general individual difference, such as for example an individual's predisposition to engage in self-care practices that overlaps with personality and mental health aspects.

Goldberg et al.'s (2018) meta-analysis indicates that the long-term effects of MBIs for depression in nonpregnant samples are superior when compared with no treatment or active control conditions. However, the specific life situation that antenatal MBI participants are in-i.e., going through the major life event of childbirth and becoming a parent shortly after the intervention has been completed-makes comparisons with MBIs among nonpregnant populations less suitable. Childbirth and the transition to parenting can have a large positive as well as negative impacts on the wellbeing of participants. This impact may thus overshadow long-term intervention effects. Furthermore, given cohort study findings that life stress and emotional stress decrease at postpartum when compared with the pregnancy period (Tegethoff et al. 2011), it is likely that in evaluations of long-term effects of antenatal MBIs, most control groups will also show improvements in psychological outcomes at postpartum.

Although data on long-term effects are sparse in the emerging research field of antenatal MBIs, some results have been published. For example, Miklowitz et al. (2015) found that lowered levels of depressive symptoms were sustained 6 months postpartum. The difference in outcome between that study and the current study may be explained by the lack of a control group in Miklowitz et al.'s study and that the sample differed from ours since it was more homogenous in regard to vulnerability but more diverse in regard to perinatal status: The participants had a history of major depressive disorder or bipolar spectrum disorder and could either be actively trying to conceive (pre-pregnant), pregnant, or in the first year postpartum. Similar to Miklowitz's study, a single-arm study by Luberto et al. (2018) showed that the improvements in anxiety found from pre- to postintervention were maintained when followed up at 3 months postpartum, and reductions in depressive symptoms found from pre- to postintervention were further reduced at 3 months postpartum. In contrast to our sample, the latter study only included pregnant women with high levels of worry and/or generalized anxiety symptoms.

On the other hand, a study by Vieten and Astin (2008) has shown that while improvements in depression and positive affect were still evident at 3 months postpartum, betweengroup changes were nonsignificant compared with a waitlist control condition. Their sample was similar to ours, with pregnant participants selected based on having previously sought some form of treatment for mood disorders. In the same manner, a pilot RCT including a sample of pregnant women experiencing high levels of perceived stress and pregnancy anxiety demonstrated larger decreases in anxiety from preto postintervention, but no sustained effects in a 6-week postintervention follow-up when comparing intervention with a reading control condition (Guardino et al. 2014).

Despite the attenuation of intervention effects in the postpartum period, our findings of significant effects at postintervention may carry benefits in their own right: The growing body of evidence of the transmission of maternal distress to offspring demonstrates that the intrauterine and early postnatal time period is a particularly sensitive developmental window (Entringer et al. 2015; Stein et al. 2014; Van Den Bergh et al. 2017). Therefore, our findings of stress and depressive symptom reduction during pregnancy corroborate that antenatal interventions are valuable. The observed increase in positive states of mind is also a valuable finding since maternal positive affect is associated with beneficial outcomes in length of gestation and reduced risk of preterm delivery (Voellmin et al. 2013). In addition, maternal mindfulness during pregnancy has been associated with better infant social-emotional development (Braeken et al. 2017). This illustrates not only the value of prevention of negative affect in pregnant mothers but also the value of the promotion of positive affect and mindfulness.

A strength of this study is the rigorous method with a randomized controlled design, an active control, and a larger sample size than in most of the previous studies, many of 
which have been uncontrolled trials or trials with non-active control groups. Since the Lamaze course is well known and appreciated in the Stockholm area where the study was conducted, it can be considered a bona fide treatment, likely to be appraised as credible and beneficial among participants.

We chose a cut-off for continued practice of mindfulness through the follow-up period, between no practice and some practice. Consequently, for some continuers, the amount of practice was very low-only a few minutes per week-and for some continuers it could be up to $200 \mathrm{~min}$ per week. Since new parents tend to be busy caring for their newborns, we reasoned that very little practice may suffice as a reminder of a mindful approach and embodiment in everyday life and may thus nevertheless be enough to sustain positive intervention effects.

\section{Limitations and Future Research}

Our study limitations include that the treatment condition was confounded by number of sessions (eight in the MBCPcondition and three in the control condition). Furthermore, in intervention studies of this type, it is not possible for participants to be blinded to treatment condition. Although a study design with an active control is more rigorous than one with no treatment control, it is likely that the active control condition also produces substantial effects. Therefore, in order to detect significant differences through the follow-up period, a larger sample size might have been needed to reduce the risk of type I error.

Most of our participants were highly educated and had medium to high household incomes, which negatively affects the generalizability of the results to other populations. When using self-report questionnaires, there is always a risk of reporting bias, which can be particularly problematic regarding the Five Facets of Mindfulness Questionnaire (Goldberg et al. 2015). Moreover, tracking continued practice of mindfulness with retrospective logs to report the length and frequency of practices is likely to result in inaccurate estimates.

Future studies could explore potential modifications of the program aiming to further strengthen the element of peer support and its continuity into the postpartum period, e.g., by offering the intervention to participants living in the same district and adding a series of booster sessions after childbirth. This may further promote the psychological wellbeing of the families since it may facilitate them to build a supportive community and continue to inspire each other to embody compassion and sensitivity in their parenting adventure.

This study gives partial support for providing MBCP for pregnant women. The differences in short- and long-term effects among the mothers in the MBCP-arm who did versus did not continue to practice mindfulness raise questions regarding susceptibility to the program. Furthermore, given that there are risks for undesirable effects from MBIs for certain individuals, under certain conditions (Britton 2019), a critical question is what characterizes mothers who can benefit from $\mathrm{MBCP}$, as well as mothers who would have more advantage from other kinds of support. A suggestion for future studies is therefore to explore what works for whom. In conclusion, despite the finding that the intervention effects were not sustained at long-term follow-up assessments during the postpartum period, the increases in psychological wellbeing found from pre- to postintervention warrant further investigations as the improvements take place in a time that is crucial for the mother-infant dyad.

Author Contributions GL collaborated in the design of the study, collected the data, carried out the analyses, and drafted the manuscript. WJ collaborated in the design of the study, supervised the analysis, and edited the manuscript. RB designed the study, supervised the analysis, and edited the manuscript. EN designed the study, supervised the collection of data, and edited the manuscript. MN designed the study, collected the data, and edited the manuscript. All authors approved the final version of the manuscript for submission.

Funding Information Open access funding provided by Karolinska Institute. This study was funded by Ekhaga Foundation (2013-32) and the Swedish Research Council (2014-10167).

Data Availability All data available on request.

\section{Compliance with Ethical Standards}

Conflict of Interest Two of the authors (GL and MN) are MBCP providers. None of the other authors report conflicts of interest.

Ethical Approval The Stockholm Regional Ethics Committee approved the study (2012/400-31/4). Written informed consent was obtained from all participants.

Open Access This article is licensed under a Creative Commons Attribution 4.0 International License, which permits use, sharing, adaptation, distribution and reproduction in any medium or format, as long as you give appropriate credit to the original author(s) and the source, provide a link to the Creative Commons licence, and indicate if changes were made. The images or other third party material in this article are included in the article's Creative Commons licence, unless indicated otherwise in a credit line to the material. If material is not included in the article's Creative Commons licence and your intended use is not permitted by statutory regulation or exceeds the permitted use, you will need to obtain permission directly from the copyright holder. To view a copy of this licence, visit http://creativecommons.org/licenses/by/4.0/.

\section{References}

Adler, N. E., Horowitz, M., Garcia, A., \& Moyer, A. (1998). Additional validation of a scale to assess positive states of mind. Psychosomatic Medicine, 60(1), 26-32.

Badker, R., \& Misri, S. (2017). Mindfulness-based therapy in the perinatal period: a review of the literature. British Columbia Medical Journal, 59(1), 18-21. 
Baer, R. A., Smith, G. T., Hopkins, J., Krietemeyer, J., \& Toney, L. (2006). Using self-report assessment methods to explore facets of mindfulness. Assessment, 13(1), 27-45. https://doi.org/10.1177/ 1073191105283504.

Bardacke, N. (2012). Mindful birthing: Training the mind, body and heart for childbirth and beyond. HarperCollins.

Bardacke, N. (2019). http://www.mindfulbirthing.org/classes-training/ mbcptt/. Accessed 23 Jan 2019.

Barkan, T., Hoerger, M., Gallegos, A. M., Turiano, N. A., Duberstein, P. R., \& Moynihan, J. A. (2016). Personality predicts utilization of mindfulness-based stress reduction during and post-intervention in a community sample of older adults. The Journal of Alternative and Complementary Medicine, 22(5), 39-395. https://doi.org/10.1089/ acm.2015.0177.

Bergström, M., Kieler, H., \& Waldenström, U. (2011). A randomised controlled multicentre trial of women's and men's satisfaction with two models of antenatal education. Midwifery, 27(6), e195-e200. https://doi.org/10.1016/j.midw.2010.07.005.

Bernstein, D., Stein, J., Newcomb, M., \& Walker, E. (2003). Development and validation of a brief screening version of the Childhood Trauma Questionnaire. Child Abuse \& Neglect, 27(2), 169-190. https://doi.org/10.1016/S0145-2134(02)00541-0.

Braeken, M. A. K. A., Jones, A., Otte, R. A., Nyklíček, I., \& Van Den Bergh, B. R. H. (2017). Potential benefits of mindfulness during pregnancy on maternal autonomic nervous system function and infant development. Psychophysiology, 54(2), 279-288. https://doi. org/10.1111/psyp.12782.

Britton, W. B. (2019). Can mindfulness be too much of a good thing? The value of a middle way. Current Opinion in Psychology, 28, 159165. https://doi.org/10.1016/j.copsyc.2018.12.011.

Cohen, S., Kamarck, T., \& Mermelstein, R. (1983). A global measure of perceived stress. Journal of Health and Social Behavour, 24(4), 385-396.

Cox, J. L., Holden, J. M., \& Sagovsky, R. (1987). Detection of postnatal depression: development of the 10- item Edinburgh Postnatal Depression Scale. The British Journal of Psychiatry, 150, 782-786.

Dhillon, A., Sparkes, E., \& Duarte, R. (2017). Mindfulness-based interventions during pregnancy: a systematic review and meta-analysis. Mindfulness, 8(6), 1421-1437. https://doi.org/10.1007/s12671-0170726-X.

Dimidjian, S., Goodman, S. H., Felder, J. N., Gallop, R., Brown, A. P., \& Beck, A. (2016). Staying well during pregnancy and the postpartum: a pilot randomized trial of mindfulness-based cognitive therapy for the prevention of depressive relapse/recurrence. Journal of Consulting and Clinical Psychology, 84(2), 134-145. https://doi. org/10.1037/ccp0000068.

Duncan, L. G., \& Bardacke, N. (2010). Mindfulness-based childbirth and parenting education: promoting family mindfulness during the perinatal period. Journal of Child and Family Studies, 19(2), 190-202. https://doi.org/10.1007/s10826-009-9313-7.

Dunn, C., Hanieh, E., Roberts, R., \& Powrie, R. (2012). Mindful pregnancy and childbirth: effects of a mindfulness-based intervention on women's psychological distress and well-being in the perinatal period. Archives of Women's Mental Health, 15(2), 139-143. https:// doi.org/10.1007/s00737-012-0264-4.

Eklund, M., Bäckström, M., \& Tuvesson, H. (2014). Psychometric properties and factor structure of the Swedish version of the Perceived Stress Scale. Nordic Journal of Psychiatry, 68(7), 494-499. https:// doi.org/10.3109/08039488.2013.877072.

Entringer, S., Buss, C., \& Wadhwa, P. D. (2015). Prenatal stress, development, health and disease risk: a psychobiological perspective2015 Curt Richter Award Paper. Psychoneuroendocrinology, 62, 366-375. https://doi.org/10.1016/j.psyneuen.2015.08.019.

Fisher, C., Hauck, Y., Bayes, S., \& Byrne, J. (2012). Participant experiences of mindfulness-based childbirth education: a qualitative study.
BMC Pregnancy and Childbirth, 12, Article 126. https://doi.org/10. 1186/1471-2393-12-126.

Fredrickson, B. L. (2001). The role of positive emotions in positive psychology. American Psychologist, 56(3), 218-226. https://doi.org/10. 1037/0003-066X.56.3.218.

Frisk, A. (2018). AnnasProfylax. Anna Frisk.

Goldberg, S. B., Wielgosz, J., Dahl, C., Schuyler, B., Maccoon, D. S., Rosenkranz, M., Lutz, A., Sebranek, C. A., \& Davidson, R. J. (2016). Does the Five Facet Mindfulness Questionnaire measure what we think it does? Construct validity evidence from an active controlled randomized clinical trial. Psychological Assessment, 28(8), 1009-10014. https://doi.org/10.1037/pas0000233 .

Goldberg, S. B., Tucker, R. P., Greene, P. A., Davidson, R. J., Wampold, B. E., Kearney, D. J., \& Simpson, T. L. (2018). Mindfulness-based interventions for psychiatric disorders: a systematic review and meta-analysis. Clinical Psychology Review, 59, 52-60. https://doi.org/ 10.1016/j.cpr.2017.10.011.

Goodman, J., Guarino, A., Chenausky, K., Klein, L., Prager, J., Petersen, R., Forget, A., \& Freeman, M. (2014). CALM pregnancy: results of a pilot study of mindfulness-based cognitive therapy for perinatal anxiety. Archives of Women's Mental Health, 17(5), 373-387. https://doi.org/10.1007/s00737-013-0402-7.

Guardino, C. M., Dunkel Schetter, C., Bower, J. E., Lu, M. C., \& Smalley, S. L. (2014). Randomised controlled pilot trial of mindfulness training for stress reduction during pregnancy. Psychology \& health., 29(3), 334-349. https://doi.org/10.1080/08870446.2013.852670.

Hall, H. G., Beattie, J., Lau, R., East, C., \& Anne Biro, M. (2016). Mindfulness and perinatal mental health: a systematic review. Women and Birth, 29(1), 62-71. https://doi.org/10.1016/j.wombi. 2015.08.006.

Hesser, H. (2015). Modeling individual differences in randomized experiments using growth models: recommendations for design, statistical analysis and reporting of results of internet interventions. Internet Interventions, 2(2), 110-120. https://doi.org/10.1016/j.invent.2015. 02.003 .

Horowitz, M., Adler, N., \& Kegeles, S. (1988). A scale for measuring the occurrence of positive states of mind: a preliminary report. Psychosomatic Medicine, 50(5), 477-483. https://doi.org/10.1097/ 00006842-198809000-00004.

Lilja, J. L., Frodi-Lundgren, A., Hanse, J. J., Josefsson, T., Lundh, L.-G., Sköld, C., Hansen, E., \& Broberg, A. G. (2011). Five Facets Mindfulness Questionnaire - reliability and factor structure: a Swedish version. Cognitive Behaviour Therapy, 40(4), 291-303. https://doi.org/10.1080/16506073.2011.580367.

Lonnberg, G., Nissen, E., \& Niemi, M. (2018). What is learned from Mindfulness Based Childbirth and Parenting Education? Participants' experiences. BMC Pregnancy and Childbirth, 18(1). https://doi.org/10.1186/s12884-018-2098-1.

Lonnberg, G., Jonas, W., Unternaehrer, E., Branstrom, R., Nissen, E., \& Niemi, M. (2019). Effects of a mindfulness based childbirth and parenting program on pregnant women's perceived stress and risk of perinatal depression-results from a randomized controlled trial. Journal of Affective Disorders, 262, 133-142. https://doi.org/10. 1016/j.jad.2019.10.048.

Luberto, C., Park, E., \& Goodman, J. (2018). Postpartum outcomes and formal mindfulness practice in mindfulness-based cognitive therapy for perinatal women. Mindfulness, 9(3), 850-859. https://doi.org/10. 1007/s12671-017-0825-8.

Mascaro, J. S., Rilling, J. K., Negi, L. T., \& Raison, C. L. (2013). Preexisting brain function predicts subsequent practice of mindfulness and compassion meditation. NeuroImage, 69(C), 35-42. https://doi. org/10.1016/j.neuroimage.2012.12.021.

Matvienko-Sikar, K., Lee, L., Murphy, G., \& Murphy, L. (2016). The effects of mindfulness interventions on prenatal well-being: a systematic review. Psychology \& Health, 31(12), 1415-1434. https:// doi.org/10.1080/08870446.2016.1220557 . 
Meyer, T., Gross, M. M., \& Roy Malis, F. (2017). Effects of an antenatal mindfulness-based childbirth and parenting programme on the postpartum experiences of mothers: a qualitative interview study. $B M C$ Pregnancy and Childbirth, 17(1). https://doi.org/10.1186/s12884017-1240-9.

Miklowitz, D., Semple, R., Hauser, M., Elkun, D., Weintraub, M., \& Dimidjian, S. (2015). Mindfulness-Based Cognitive Therapy for perinatal women with depression or bipolar spectrum disorder. Cognitive Therapy and Research, 39(5), 590-600. https://doi.org/ 10.1007/s10608-015-9681-9.

Rubertsson, B., Berglund, J., \& Sydsjö. (2011). The Swedish validation of Edinburgh Postnatal Depression Scale (EPDS) during pregnancy. Nordic Journal of Psychiatry, 65(6), 414-418. https://doi.org/10. 3109/08039488.2011.590606.

Shi, Z., \& MacBeth, A. (2017). The effectiveness of Mindfulness-Based Interventions on maternal perinatal mental health outcomes: a systematic review. Mindfulness, 8(4), 823-847. https://doi.org/10.1007/ s12671-016-0673-y.

Stein, A., Pearson, R. M., Goodman, S. H., Rapa, E., Rahman, A., McCallum, M., Howard, L. M., \& Pariante, C. M. (2014). Effects of perinatal mental disorders on the fetus and child. Lancet, 384(9956), 1800-1819. https://doi.org/10.1016/s0140-6736(14) 61277-0.

Tegethoff, M., Greene, N., Olsen, J., Schaffner, E., \& Meinlschmidt, G. (2011). Stress during pregnancy and offspring pediatrie disease: a national cohort study. Environmental Health Perspectives, 119(11), 1647-1652. https://doi.org/10.1289/ehp.1003253.

Townshend, K., Caltabiano, N., Powrie, R., \& O'Grady, H. (2018). A preliminary study investigating the effectiveness of the caring for body and mind in pregnancy (CBMP) in reducing perinatal depression, anxiety and stress. Journal of Child and Family Studies, 27(5), 1556-1566. https://doi.org/10.1007/s10826-017-0978-z.

Van Den Bergh, B. R. H., van Den Heuvel, M. I., Lahti, M., Braeken, M., de Rooij, S. R., Entringer, S., Hoyer, D., Roseboom, T., Räikkönen, K., King, S., \& Schwab, M. (2017). Prenatal developmental origins of behavior and mental health: the influence of maternal stress in pregnancy. Neuroscience and Biobehavioral Reviews. S0149-
7634(16)30734-5. https://doi.org/10.1016/j.neubiorev.2017.07.003

Vieten, C., \& Astin, J. (2008). Effects of a mindfulness-based intervention during pregnancy on prenatal stress and mood: results of a pilot study. Archives of Womens Mental Health, 11(1), 67-74. https:// doi.org/10.1007/s00737-008-0214-3.

Voellmin, A., Entringer, S., Moog, N., Wadhwa, P. D., \& Buss, C. (2013). Maternal positive affect over the course of pregnancy is associated with the length of gestation and reduced risk of preterm delivery. Journal of Psychosomatic Research, 75(4), 336-340. https://doi. org/10.1016/j.jpsychores.2013.06.031.

Warriner, S., Crane, C., Dymond, M., \& Krusche, A. (2018). An evaluation of mindfulness-based childbirth and parenting courses for pregnant women and prospective fathers/partners within the UK NHS (MBCP-4-NHS). Midwifery, 64, 1-10. https://doi.org/10. 1016/j.midw.2018.05.004.

WHO. (2019a). Gender and women's mental health. World Health Organization Retrieved 08.21 from https://www.who.int/mental health/prevention/genderwomen/en. Accessed 21 Aug 2019.

WHO. (2019b). Maternal and child mental health. WHO. Retrieved May 2019 from https://www.who.int/mental_health/maternalchild/en/. Accessed May 2019.

Wickberg, B., \& Hwang, C. P. (1996). The Edinburgh Postnatal Depression Scale: validation on a Swedish community sample. Acta Psychiatrica Scandinavica, 94(3), 181-184. https://doi.org/ 10.1111/j.1600-0447.1996.tb09845.x.

Woolhouse, H., Mercuri, K., Judd, F., \& Brown, S. J. (2014). Antenatal mindfulness intervention to reduce depression, anxiety and stress: a pilot randomised controlled trial of the MindBabyBody program in an Australian tertiary maternity hospital. BMC Pregnancy and Childbirth, 14, 16, article 369. https://doi.org/10.1186/s12884-0140369-z.

Publisher's Note Springer Nature remains neutral with regard to jurisdictional claims in published maps and institutional affiliations. 